\section{Assistência à saúde mental no Brasil: estudo multifacetado em quatro grandes cidades}

\author{
Mental healthcare in Brazil: a multifaceted study \\ in four large cities
}

\section{Asistencia en salud mental en Brasil: estudio multifacético en cuatro grandes ciudades}

Carlos Eduardo Menezes Amaral 1

Carlos Alberto dos Santos Treichel 2

Priscila Maria Stolses Bergamo Francisco ${ }^{2}$

Rosana Teresa Onocko-Campos ${ }^{2}$

\section{Resumo}

O objetivo deste estudo foi avaliar as características das redes de saúde de quatro grandes municipios brasileiros (Campinas, Fortaleza, Porto Alegre e São Paulo) no que diz respeito à prestação de cuidados em saúde mental. Foram usados como desfechos: (i) local de identificação do problema de saúde mental; (ii) atendimento em saúde mental na atenção básica; (iii) assistência farmacêutica em saúde mental; e (iv) reinserção social. Trata-se de um estudo analítico de métodos mistos, de abordagem concomitante e sequencial, conduzido com 10 gestores e 1.642 usuários de Centros de Atenção Psicossocial (CAPS) dos municípios citados. Observou-se a persistência de serviços de alta complexidade, tais como os hospitais, como locais de identificação inicial dos problemas de saúde mental em Campinas (40\% dos usuários) e Fortaleza (37\%); baixa proporção de tratamento de saúde mental na atenção básica (Fortaleza, 23\%); diferenças entre os municípios no que diz respeito à prescrição de medicamentos psicotrópicos na atenção básica (Porto Alegre, 68\%; São Paulo, 64\%; Campinas, 39\%; Fortaleza, 31\%), bem como na falta dos medicamentos prescritos (maior em Fortaleza, 58\%; menor em Campinas, 28\%); e fragilidade em geral na retomada de atividades laborais (menor em São Paulo, 17\%; maior em Campinas, 39\%), havendo melhores resultados em geral em relação a atividades religiosas e de lazer (maiores em São Paulo, 53\% e 56\%, respectivamente). É um estudo que contribui para a discussão do panorama brasileiro da assistência à saúde mental, com evidências da persistência de desigualdades no contexto nacional, e aponta lacunas em algumas configurações das redes de saúde mental com potenciais para melhor desempenho e seguimento longitudinal.

Atenção Básica; Atenção Secundária à Saúde; Serviços Comunitários de Saúde Mental; Rede de Cuidados Continuados de Saúde;

Métodos de Avaliação

\author{
Correspondência \\ C. E. M. Amaral \\ Colegiado de Medicina de Paulo Afonso, Universidade Federal \\ do Vale do São Francisco. \\ Rua da Aurora s/n, Paulo Afonso, BA 48607-190, Brasil. \\ caduamaral@gmail.com \\ 1 Colegiado de Medicina de Paulo Afonso, Universidade Federal \\ do Vale do São Francisco, Paulo Afonso, Brasil. \\ 2 Escola de Ciêncas Médicas, Universidade Estadual de \\ Campinas, Campinas, Brasil.
}




\section{Introdução}

Atualmente, estima-se que os transtornos mentais são responsáveis por $30 \%$ da carga de doenças não fatais e $10 \%$ da carga total de doenças no mundo 1 . Esses transtornos têm um impacto significativo na saúde pública e resultam em elevadas taxas de incapacidade e mortalidade entre pessoas convivendo com essas condições 2 .

O suicídio é a segunda causa mais frequente de morte entre a população jovem em nível mundial 3. Indivíduos com transtornos mentais em todas as faixas de idade estão sujeitos a uma importante disparidade em saúde. Devido a problemas físicos/clínicos que não são devidamente assistidos, entre eles hipertensão e diabetes, pessoas com quadros de depressão ou esquizofrenia apresentam uma probabilidade $40 \%$ a 60\% maior de morte prematura quando comparadas à população em geral 3 .

Frente a essa perspectiva, nos últimos anos a Organização Mundial da Saúde (OMS) definiu o enfrentamento da diferença de mortalidade dos portadores de transtornos mentais como um dos desafios do milênio 1,2. Entre as ações recomendadas para esse fim estão a expansão e o fortalecimento dos serviços comunitários de saúde mental e o fomento do cuidado em saúde mental na atenção básica (AB), para o aumento da capacidade de identificação e manejo dos casos neste ponto de atenção. Nesse contexto, a provisão de tratamento medicamentoso adequado e a promoção de atividades que estimulem a inserção e a reabilitação psicossocial dos usuários são consideradas elementos fundamentais para as linhas de cuidado 4,5 .

Ressalta-se que nas duas últimas décadas o Brasil expandiu sua rede de serviços especializados comunitários e atualmente o país conta com mais de 2.300 Centros de Atenção Psicossocial (CAPS) nos quais trabalham mais de 30 mil profissionais 6 . Entretanto, apesar dessa expansão desses serviços, ainda são escassos os estudos que se propõem a uma avaliação abrangente do impacto e das relações entre os serviços das redes de saúde.

A expansão dos serviços comunitários de saúde mental aconteceu de forma desigual, resultando no estabelecimento de redes heterogêneas em diferentes cidades e regiões 7,8,9. Dessa forma, as pesquisas sobre avaliação ou análise das políticas públicas de saúde mental têm sido instigadas a alargar seus referenciais teóricos e utilizar estratégias capazes de acolher a diversidade do campo.

Nesse sentido, este trabalho buscou avaliar as redes de saúde mental de quatro grandes municípios brasileiros: Campinas (São Paulo), Fortaleza (Ceará), Porto Alegre (Rio Grande do Sul) e São Paulo. Para isso, foram elencados como principais desfechos de interesse o local de identificação inicial do problema de saúde mental, a oferta de cuidados em saúde mental na $\mathrm{AB}$, a assistência farmacêutica na $\mathrm{AB}$ e marcadores do processo de reabilitação social relacionados a trabalho, lazer e religião.

\section{Metodologia}

\section{Tipo de estudo e delineamento}

Trata-se de um estudo analítico de métodos mistos, de abordagem concomitante e sequencial 10, conduzido com usuários de CAPS dos municípios citados. O trabalho é um recorte de uma pesquisa maior que analisou o acesso aos serviços com base na $\mathrm{AB}$, além do funcionamento e utilização da atenção especializada (AE) para hipertensão arterial grave, gravidez de alto risco, câncer de mama e transtornos graves de saúde mental.

Elaborou-se nesta pesquisa um modelo teórico-analítico que lançou mão das seguintes estratégias de investigação: (i) análise de dados secundários; (ii) estudo qualitativo descritivo; e (iii) estudo transversal realizado com base em um inquérito nos serviços de saúde. Uma discussão em profundidade acerca da metodologia encontra-se disponível em outra publicação ${ }^{11}$.

Neste trabalho, apresentamos os resultados da triangulação entre o estudo qualitativo acerca do funcionamento de cada rede municipal de serviços e do estudo quantitativo, transversal, conduzido com usuários dos CAPS de cada município. 


\section{População e seleção dos participantes}

No estudo qualitativo foi realizada amostragem intencional, de forma a contemplar os gestores de cada rede municipal de saúde. Foram entrevistados de 3 a 5 gestores de cada município, que atuavam na coordenação de saúde mental (ou órgão equivalente) ou na gestão local de serviços CAPS, totalizando 10 informantes. Os entrevistados foram selecionados com base nas posições que ocupavam na rede de saúde, de forma a possuírem, por sua experiência de trabalho, informações precisas em relação à regulação em cada município e à história recente da rede.

Para o estudo transversal foi considerada como população do estudo o conjunto de usuários atendidos nos CAPS destinados ao acompanhamento de adultos com transtornos mentais graves, nos municípios da pesquisa. Não foram considerados no estudo os CAPS Infanto-Juvenil e CAPS AD (álcool e outras drogas).

Nos municípios de Campinas, Fortaleza e Porto Alegre, o trabalho foi realizado em todos os serviços disponíveis: seis CAPS III em Campinas; cinco CAPS II e um CAPS III em Fortaleza; quatro CAPS II em Porto Alegre. No Município de São Paulo, que durante a realização da pesquisa contava com 34 serviços, optou-se por questões logísticas pela inclusão dos 24 CAPS com maior volume de usuários.

Os critérios de inclusão foram: ter iniciado o tratamento no serviço há menos de três anos; residir no município do serviço; apresentar condições cognitivas de responder ao questionário; e não estar em crise, hospitalização psiquiátrica ou hospitalidade integral em CAPS III durante o período da pesquisa.

A seleção dos participantes foi feita por amostragem não probabilística. Os usuários que frequentaram o serviço no período da coleta foram acessados, e nos casos em que preenchiam o critério de inclusão, convidados a participar do trabalho.

\section{$\underline{\text { Logística }}$}

Para o estudo qualitativo foram realizadas entrevistas semiestruturadas com informantes-chave das Secretarias Municipais de Saúde de cada município. Essa abordagem permitiu levantar informações acerca da estrutura e funcionamento das redes de cada município, caracterizando-as de acordo com sua regulação e fluxo entre os serviços dos diferentes níveis de atenção.

Para o estudo quantitativo foram realizadas aplicações de questionários nos serviços estudados entre março e setembro de 2016, por meio de um aplicativo contendo o questionário da pesquisa. As aplicações do questionário ocorreram nos dias em que os usuários compareciam ao serviço para atendimento ou outra atividade terapêutica e foram realizadas por entrevistadores previamente treinados.

\section{Controle de qualidade}

A entrada dos dados no banco foi feita por meio do aplicativo de questionários, automaticamente convertidos em planilhas. O controle de qualidade foi realizado pelos supervisores de campo com checagem diária da consistência dos dados. Inconsistências foram avaliadas e corrigidas quando necessário.

\section{Desfechos}

Foram considerados desfechos de interesse no estudo transversal: local de identificação do problema de saúde mental; recebimento de atendimento de saúde mental na $\mathrm{AB}$; prescrição de medicamentos psicotrópicos na $\mathrm{AB}$; falta de medicações psicotrópicas na farmácia nos últimos seis meses; início ou retomada das atividades de trabalho, lazer e religião. Para o desfecho "local de identificação do problema de saúde mental", considerou-se "identificação" como o primeiro apontamento, por um profissional de saúde, da necessidade de avaliação, tratamento ou acompanhamento de um agravo de saúde mental. 


\section{Covariáveis}

Foram identificadas algumas características sociodemográficas dos usuários e interação com a AB. As variáveis incluídas foram: sexo (masculino, feminino); cor (branca, preta, parda); idade, em anos (18 a 30, 31 a 40, 41 a 50, 51 ou mais); escolaridade, em anos de estudo (até 4, 5 a 9, 10 a 13, 14 ou mais); recebimento de tratamento em saúde mental na $\mathrm{AB}$ (não recebeu, recebeu); referência do caso para $\mathrm{AB}$ (não, sim).

\section{Análises estatísticas}

As análises foram realizadas com o pacote estatístico Stata 11 (https://www.stata.com). Inicialmente, utilizou-se estatística descritiva para caracterizar a amostra de cada município. Por serem variáveis categóricas, utilizou-se o teste qui-quadrado para identificar diferenças significativas entre as proporções apresentadas em cada município. A significância estatística foi definida como valor de $\mathrm{p}<0,05$.

\section{Procedimentos éticos}

O trabalho foi submetido e aprovado pelo Comitê de Ética em Pesquisa da Universidade Estadual de Campinas (parecer no 1.100.984). A condução do estudo seguiu as diretrizes de regulamentação de pesquisas envolvendo seres humanos (Resolução no 466/2012, do Conselho Nacional de Saúde), além das disposições da Declaração de Helsínque.

\section{Resultados}

\section{Componente qualitativo: características de funcionamento das redes de saúde mental de cada município estudado}

Baseando-se nas entrevistas, foi possível sistematizar informações contextuais sobre a regulação de cada rede. A forma de acesso ao CAPS, assim como a quantidade de equipes de apoio e disponibilidade de profissionais, apresentam variações importantes em cada cidade.

Campinas tem ampla cobertura de CAPS III, contando com seis serviços para uma população de aproximadamente um milhão de habitantes. O acesso aos CAPS ocorre tanto por demanda espontânea como via encaminhamento da $\mathrm{AB}$. O município tem forte tradição de apoio matricial na $\mathrm{AB}$, especialmente no campo da saúde mental, com a presença de equipes de apoio em saúde mental anterior à criação da política do Núcleo de Apoio à Saúde da Família (NASF).

As equipes originais de apoio em saúde mental contam com psiquiatra e um segundo profissional de psicologia ou terapia ocupacional, fornecendo apoio às equipes da Estratégia Saúde da Família (ESF) de uma mesma unidade de saúde. Contudo, no município essas equipes têm sido progressivamente substituídas por equipes do modelo NASF, responsáveis por um quantitativo maior de equipes e unidades. Adicionalmente, no ano de 2012, uma mudança na forma de contratação de psiquiatras na $\mathrm{AB}$ (originalmente via uma organização não governamental conveniada com o poder público) ocasionou um esvaziamento desta categoria na $\mathrm{AB}$ do município, seguida por um lento processo de substituição via contratação direta pela administração pública.

No Município de Fortaleza, os serviços CAPS também funcionam no modelo "portas abertas", sendo comuns tanto acesso direto como via encaminhamento. A capital cearense apresenta fragilidades na manutenção de equipes NASF, uma vez que no período pesquisado havia apenas uma equipe por Secretaria Executiva Regional (divisões administrativas que têm em média cerca de 400 mil habitantes). Além disso, não há psiquiatras nas equipes NASF, tornando o seu acesso no Sistema Único de Saúde (SUS) possível apenas na rede especializada.

Fortaleza conta com um hospital psiquiátrico estadual, que oferece, além de internação e hospital-dia, pronto-atendimento e atendimento ambulatorial, e concentra grande parte dos psiquiatras da rede pública. Em relação aos atendimentos de psicologia, a rede municipal (tanto AB como CAPS) com 
frequência necessita realizar encaminhamentos para os serviços de clínica-escola das universidades do município, em função da demanda excedente.

Em São Paulo, o acesso ao CAPS é possível via demanda espontânea ou encaminhamento. O município tem equipes NASF/apoio matricial com a presença de psiquiatras, psicólogos e terapeutas ocupacionais. Observa-se dificuldade da manutenção dos psiquiatras na equipe, ocorrendo grande rotatividade, em especial na periferia. Prevê-se uma equipe de apoio por grupo de 5 a 8 equipes de saúde da família, mas na prática apenas cerca de $60 \%$ das equipes contam com cobertura NASF. A diversidade dos tipos, modelos de gestão e contratos dos serviços de AB dificulta a adoção de um modelo padrão de encaminhamento, o que pode aumentar a busca por acesso direto ao CAPS, sem referenciamento por outros serviços da rede.

Na cidade de Porto Alegre, o protocolo de acesso ao CAPS prevê o encaminhamento de equipes de matriciamento, havendo raras exceções de demanda espontânea no serviço. Dessa forma, a quase totalidade de usuários do CAPS tem sido encaminhada pela AB. Os atendimentos individuais em saúde mental não se configuram como atividade prioritária das equipes de matriciamento/NASF do município, sendo realizados por apenas alguns dos profissionais que as compõem. Algumas das equipes de matriciamento/NASF têm psiquiatras em sua configuração, porém observa-se a migração de psiquiatras para a rede especializada, em especial os hospitais, que vêm expandindo sua atuação na rede de atenção em saúde do município. O Quadro 1 apresenta uma síntese do levantamento realizado.

\section{Quadro 1}

Características das redes de saúde mental dos municípios estudados.

\begin{tabular}{|c|c|c|c|c|}
\hline & CAMPINAS & FORTALEZA & PORTO ALEGRE & SÃO PAULO \\
\hline $\begin{array}{l}\text { Modelo de acesso aos } \\
\text { CAPS }\end{array}$ & $\begin{array}{c}\text { Modelo "portas } \\
\text { abertas"; contam com } \\
\text { ações de referência- } \\
\text { contrarreferência via } \\
\text { NASF e equipes de saúde } \\
\text { mental na AB }\end{array}$ & $\begin{array}{c}\text { Modelo "portas } \\
\text { abertas"; contam com } \\
\text { ações de referência- } \\
\text { contrarreferência via } \\
\text { NASF e equipes de saúde } \\
\text { mental na AB }\end{array}$ & $\begin{array}{c}\text { Modelo "portas } \\
\text { abertas"; contam com } \\
\text { ações de referência- } \\
\text { contrarreferência via } \\
\text { NASF e equipes de saúde } \\
\text { mental na AB }\end{array}$ & $\begin{array}{l}\text { Modelo "portas abertas" } \\
\text { e contam com ações } \\
\text { de matriciamento e } \\
\text { referência via NASF }\end{array}$ \\
\hline $\begin{array}{l}\text { Presença de equipes } \\
\text { NASF e/ou outros } \\
\text { formatos de apoio } \\
\text { matricial }\end{array}$ & $\begin{array}{l}\text { Existem } 6 \text { equipes NASF } \\
\text { em funcionamento no } \\
\text { município e equipes } \\
\text { de apoio em saúde } \\
\text { mental. Os CAPS } \\
\text { também realizam ações } \\
\text { sistematizadas de } \\
\text { matriciamento junto às } \\
\text { UBS }\end{array}$ & $\begin{array}{c}\text { Existem } 6 \text { equipes } \\
\text { NASF que cobrem } \\
\text { aproximadamente } 20 \% \\
\text { das equipes da ESF do } \\
\text { município }\end{array}$ & $\begin{array}{l}\text { Existem } 8 \text { equipes NASF } \\
\text { e } 1 \text { Núcleo de Apoio } \\
\text { Matricial de Atenção } \\
\text { Básica }\end{array}$ & $\begin{array}{c}\text { Existem } 100 \text { equipes } \\
\text { NASF que cobrem entre } 5 \\
\text { e } 8 \text { equipes da ESF cada }\end{array}$ \\
\hline $\begin{array}{l}\text { Composição das equipes } \\
\text { NASF e/ou de apoio em } \\
\text { saúde mental }\end{array}$ & $\begin{array}{c}\text { Presença de psicólogos, } \\
\text { psiquiatras e terapeutas } \\
\text { ocupacionais }\end{array}$ & $\begin{array}{c}\text { Presença principalmente } \\
\text { de psicólogos, porém } \\
\text { pequena quantidade de } \\
\text { equipes. Ausência de } \\
\text { psiquiatra e presença } \\
\text { eventual de terapeuta } \\
\text { ocupacional }\end{array}$ & $\begin{array}{l}\text { Presença de pelo menos } \\
\text { um profissional de saúde } \\
\text { mental das categorias } \\
\text { psicólogo ou psiquiatra, } \\
\text { além de terapeutas } \\
\text { ocupacionais }\end{array}$ & $\begin{array}{c}\text { Presença de psicólogos, } \\
\text { psiquiatras e terapeutas } \\
\text { ocupacionais }\end{array}$ \\
\hline
\end{tabular}

AB: atenção básica; CAPS: Centro de Atenção Psicossocial; ESF: Estratégia Saúde da Família; NASF: Núcleo de Apoio à Saúde da Família; UBS: unidade básica de saúde.

Fonte: elaboração própria. 


\section{Perfil dos usuários com base no estudo transversal}

Foi realizada a aplicação de questionário a 1.642 usuários de CAPS dos municípios estudados. A quantidade de usuários por município foi: 393 em Campinas; 601 em Fortaleza; 351 em Porto Alegre; e 297 em São Paulo. As características sociodemográficas podem ser observadas na Tabela 1, que dispõe dados acerca do sexo, cor, idade e escolaridade dos participantes.

\section{Local de identificação do problema de saúde mental}

Entendendo o local de identificação do problema de saúde mental como um importante marcador do funcionamento das redes de cuidado, buscou-se levantar o cenário onde um profissional de saúde havia identificado pela primeira vez a existência de um problema de saúde mental entre os usuários. Na Tabela 2 observam-se os resultados de cada município.

É possível apontar diferenças importantes nas proporções observadas para cada município quanto ao local de identificação do problema de saúde mental. Em São Paulo e Porto Alegre houve maior ocorrência de indivíduos que tiveram o seu problema identificado em uma unidade básica de saúde (UBS) - 54,3\% e 37\%, respectivamente -, já em Campinas e Fortaleza há uma parcela expressiva de usuários que foram identificados no CAPS, em hospital geral ou psiquiátrico.

Em Campinas, 25,5\% dos usuários tiveram o seu problema de saúde mental identificado no CAPS. Houve ainda ocorrência expressiva de usuários identificados em ambientes hospitalares: $21 \% \mathrm{em}$ hospital geral e 18,8\% em hospital psiquiátrico.

No Município de Fortaleza, foi observada maior proporção de usuários que tiveram o seu problema identificado em hospitais psiquiátricos (36,7\%). Na cidade de São Paulo, apenas $8 \%$ dos usuários relataram esse tipo de serviço como o primeiro local de identificação do problema.

A identificação do problema de saúde mental em ambientes hospitalares foi relatada de forma expressiva ainda entre os usuários de Porto Alegre, onde 16,4\% deles referiram ter sido identificados em hospitais gerais e 15,5\% em hospitais psiquiátricos. Ressalta-se que os hospitais gerais também foram prevalentes no Município de São Paulo (15,2\%).

\section{Tabela 1}

Caracterização sociodemográfica dos usuários incluídos no estudo, de acordo com o município estudado.

\begin{tabular}{|c|c|c|c|c|c|c|c|c|}
\hline \multirow[t]{2}{*}{ Características } & \multicolumn{2}{|c|}{ Campinas } & \multicolumn{2}{|c|}{ Fortaleza } & \multicolumn{2}{|c|}{ Porto Alegre } & \multicolumn{2}{|c|}{ São Paulo } \\
\hline & $\mathbf{n}$ & $\%$ & $\mathbf{n}$ & $\%$ & $\mathbf{n}$ & $\%$ & $\mathbf{n}$ & $\%$ \\
\hline \multicolumn{9}{|l|}{ Sexo } \\
\hline Feminino & 175 & 44,9 & 373 & 62,9 & 192 & 54,7 & 185 & 62,3 \\
\hline Masculino & 215 & 55,1 & 220 & 37,1 & 159 & 45,3 & 112 & 37,7 \\
\hline \multicolumn{9}{|l|}{ Cor } \\
\hline Branca & 171 & 44,3 & 156 & 26,4 & 213 & 61,6 & 144 & 48,8 \\
\hline Preta & 69 & 17,9 & 64 & 10,8 & 55 & 15,9 & 34 & 11,5 \\
\hline Parda & 146 & 37,8 & 371 & 62,8 & 78 & 22,5 & 117 & 39,7 \\
\hline \multicolumn{9}{|l|}{ Idade (anos) } \\
\hline $18-30$ & 92 & 24,0 & 119 & 21,1 & 63 & 18,3 & 54 & 18,1 \\
\hline $31-40$ & 100 & 26,1 & 117 & 20,7 & 72 & 20,9 & 75 & 25,2 \\
\hline $41-50$ & 82 & 21,4 & 127 & 22,5 & 96 & 27,9 & 85 & 28,6 \\
\hline 51 ou mais & 109 & 28,4 & 200 & 35,5 & 113 & 32,8 & 83 & 27,9 \\
\hline \multicolumn{9}{|c|}{ Escolaridade (anos de estudos) } \\
\hline Até 4 & 77 & 19,8 & 170 & 28,9 & 65 & 18,5 & 64 & 21,7 \\
\hline $5-9$ & 128 & 32,9 & 153 & 26,0 & 87 & 24,8 & 96 & 32,5 \\
\hline $10-13$ & 152 & 39,0 & 205 & 34,8 & 149 & 42,5 & 109 & 36,9 \\
\hline 14 ou mais & 32 & 8,2 & 60 & 10,2 & 49 & 14,0 & 26 & 8,8 \\
\hline
\end{tabular}

Fonte: elaboração própria. 
Tabela 2

Local de identificação do problema de saúde mental dos usuários incluídos no estudo, de acordo com o município estudado.

\begin{tabular}{|c|c|c|c|c|c|c|c|c|}
\hline \multirow[t]{2}{*}{ Local } & \multicolumn{2}{|c|}{ Campinas } & \multicolumn{2}{|c|}{ Fortaleza } & \multicolumn{2}{|c|}{ Porto Alegre } & \multicolumn{2}{|c|}{ São Paulo } \\
\hline & $\mathbf{n}$ & $\%$ & $\mathbf{n}$ & $\%$ & $\mathbf{n}$ & $\%$ & $\mathbf{n}$ & $\%$ \\
\hline UBS & 68 & 18,3 & 104 & 17,8 & 120 & 37,0 & 157 & 54,3 \\
\hline CAPS & 94 & 25,5 & 97 & 16,6 & 26 & 8,0 & 34 & 11,8 \\
\hline Ambulatório & 9 & 2,4 & 4 & 0,7 & 24 & 7,4 & 7 & 2,4 \\
\hline Hospital geral & 78 & 21,0 & 58 & 9,9 & 53 & 16,4 & 44 & 15,2 \\
\hline Hospital psiquiátrico & 70 & 18,8 & 215 & 36,7 & 47 & 15,5 & 23 & 8,0 \\
\hline Pronto-socorro & 15 & 4,0 & 11 & 1,9 & 10 & 3,1 & 6 & 2,1 \\
\hline Judiciário & 0 & 0,0 & 0 & 0,0 & 37 & 11,4 & 0 & 0,0 \\
\hline Outro serviço & 38 & 10,2 & 96 & 16,4 & 7 & 2,2 & 18 & 6,2 \\
\hline
\end{tabular}

CAPS: Centro de Atenção Psicossocial; UBS: unidade básica de saúde.

Fonte: elaboração própria.

\section{Tratamento de saúde mental na atenção básica}

Considerando a ação da $\mathrm{AB}$ no cuidado em saúde mental como essencial para o cuidado articulado em rede, buscou-se identificar a proporção de usuários que recebeu intervenções de saúde mental neste ponto da rede.

Considerou-se como atendimento de saúde mental qualquer tipo de tratamento, não discriminando tipo de profissional ou procedimento realizado. Inclui, portanto, prescrição de psicofármacos, psicoeducação, psicoterapia breve e grupos terapêuticos.

No Município de Campinas, 44,6\% $(\mathrm{n}=156)$ dos usuários referiram ter recebido algum tratamento de saúde mental na AB. Em Fortaleza, a parcela de usuários que havia recebido esse tipo de atendimento correspondeu a 23,3\% $(\mathrm{n}=102)$. Já em Porto Alegre e São Paulo, correspondeu a 53,6\% $(\mathrm{n}=185)$ e $56,4 \%(\mathrm{n}=154)$, respectivamente.

\section{Prescrição de medicamentos psicotrópicos na atenção básica}

Compreendendo a prescrição de medicamentos psicotrópicos como um dos marcadores da ação dos serviços de $\mathrm{AB}$ no cuidado aos usuários com problemas graves de saúde mental, buscou-se levantar a proporção de usuários que receberam este tipo de prescrição em uma UBS em cada um dos municípios estudados.

No Município de Campinas, 39,3\% ( $\mathrm{n}=137)$ dos usuários referiram ter recebido alguma prescrição de medicação para o seu problema de saúde mental na AB. Já em Fortaleza, a parcela de usuários que havia recebido esse tipo de prescrição na $\mathrm{AB}$ foi de $31,3 \%(\mathrm{n}=137)$.

Porto Alegre e São Paulo foram os municípios onde, proporcionalmente, mais usuários receberam prescrição de medicações psicotrópicas por um médico da $\mathrm{AB}$. Nesses municípios, a proporção de usuários que referiu esse tipo de ação foi de 68,2\% (n=234) e 64\% (n=174), respectivamente.

\section{Falta de medicamentos}

A fim de desvelar aspectos do funcionamento das redes acerca do fornecimento de medicações, buscou-se identificar a proporção de usuários que tiveram problemas em obter medicações prescritas nos seis meses que antecederam a aplicação do questionário, em função da falta de medicamentos na farmácia utilizada. A Tabela 3 apresenta os resultados em cada município.

Foi possível observar uma importante diferença entre os municípios no que diz respeito à disponibilidade dos medicamentos prescritos. Em Campinas, 71,5\% dos usuários relataram não haver 
Tabela 3

Falta de medicamentos prescritos na farmácia utilizada pelos usuários, de acordo com o município estudado.

\begin{tabular}{lcccccccc} 
& \multicolumn{2}{c}{ Campinas } & \multicolumn{2}{c}{ Fortaleza } & \multicolumn{2}{c}{ Porto Alegre } & \multicolumn{2}{c}{ São Paulo } \\
& $\mathbf{n}$ & $\mathbf{0}$ & $\mathbf{n}$ & $\mathbf{\%}$ & $\mathbf{n}$ & $\mathbf{\%}$ & $\mathbf{n}$ & $\%$ \\
\hline Não faltou & 276 & 71,5 & 158 & 28,1 & 168 & 49,1 & 178 & 61,8 \\
Faltou uma vez & 62 & 16,1 & 74 & 13,1 & 38 & 11,1 & 49 & 17,0 \\
Faltou mais de uma vez & 48 & 12,4 & 331 & 58,8 & 136 & 39,8 & 61 & 21,2 \\
\hline
\end{tabular}

Fonte: elaboração própria.

falta de medicações na farmácia utilizada, já em Fortaleza e Porto Alegre, 58,8\% e 39,8\% dos usuários, respectivamente, relataram problemas para obter as medicações prescritas mais de uma vez, nos últimos seis meses.

No Município de São Paulo, embora a maioria dos usuários $(61,8 \%)$ tenha relatado que não houve falta de medicamentos na farmácia utilizada por eles nos últimos seis meses, uma parcela expressiva deles $(21,2 \%)$ relatou falta de medicamentos em mais de uma ocasião no período estudado.

\section{Início ou retomada das atividades de trabalho, lazer e religião}

Entendendo o início ou retomada das atividades de trabalho, lazer e religião após a entrada do usuário no CAPS como importante indicador do processo de reinserção social, buscou-se inicialmente rastrear a proporção de usuários que apresentavam problemas relacionados a tais atividades, bem como a proporção deles que conseguiu iniciar ou retomar as atividades após a sua entrada no serviço. A quantidade de usuários que iniciou ou retomou atividades relacionadas a trabalho, lazer e religião em cada município pode ser observada na Tabela 4.

Quanto às atividades de trabalho, a maior proporção de usuários que iniciou ou retomou as atividades após a sua entrada no serviço foi observada em Campinas (39,6\%). Por outro lado, a menor proporção foi encontrada no Município de São Paulo, onde 17,3\% deles que não trabalhavam ou tiveram problemas no trabalho iniciaram ou retomaram essas atividades.

Já para as atividades de lazer, os usuários do Município de São Paulo que haviam manifestado problemas antes de sua entrada no serviço foram os que apresentaram maior retomada deste tipo de atividade (56,5\%), em comparação com os outros municípios. Em seguida destacam-se Campinas e Porto Alegre, ambos com uma proporção de aproximadamente 51\%. A menor quantidade foi observada em Fortaleza, onde 37,8\% dos usuários que relataram problemas com lazer conseguiram retomar as atividades.

No que diz respeito às atividades religiosas, os usuários de São Paulo também foram os que, comparativamente, apresentaram maior início ou retomada de atividades (53,3\%). Em Campinas, foi observada a menor proporção de sucesso nesse indicador (38,3\%).

\section{Discussão}

Estudos conduzidos anteriormente avaliaram aspectos como efetividade ${ }^{12}$, resultados 13 e satisfação 14 com os CAPS, contudo, estes constituem estudos regionais e não destacam as relações destes serviços com outros pontos da rede de atenção. Dessa forma, a inovação do presente trabalho repousa no destaque à interface entre estrutura e funcionamento da rede de saúde mental, particularmente no que compete à relação entre $\mathrm{AB}$ e $\mathrm{AE}$ em quatro grandes cidades, em três regiões do país.

Nesse sentido, os primeiros resultados ressaltados são a heterogeneidade e as diferenças de funcionamento da rede de serviços de saúde mental encontradas no estudo. Esse achado corrobora perspectivas apontadas em trabalho prévio 8 , apontando diferenças no perfil de usuários atendidos 
Prevalência de retomada das atividades de trabalho, lazer e religião entre os usuários que referiram problemas nestas áreas antes da entrada no Centro de Atenção Psicossocial (CAPS), de acordo com o município estudado.

\begin{tabular}{|c|c|c|c|c|c|c|c|c|}
\hline & \multicolumn{2}{|c|}{ Campinas } & \multicolumn{2}{|c|}{ Fortaleza } & \multicolumn{2}{|c|}{ Porto Alegre } & \multicolumn{2}{|c|}{ São Paulo } \\
\hline & $\mathbf{n}$ & $\%$ & $\mathbf{n}$ & $\%$ & $\mathbf{n}$ & $\%$ & $\mathbf{n}$ & $\%$ \\
\hline \multicolumn{9}{|c|}{ Início ou retomada das atividades de trabalho } \\
\hline Sim & 122 & 39,6 & 122 & 24,1 & 108 & 36,0 & 38 & 17,3 \\
\hline Não & 186 & 60,4 & 384 & 75,9 & 192 & 64,0 & 181 & 82,7 \\
\hline \multicolumn{9}{|c|}{ Início ou retomada das atividades de lazer } \\
\hline Sim & 120 & 51,6 & 170 & 37,8 & 134 & 51,5 & 104 & 56,5 \\
\hline Não & 116 & 48,4 & 280 & 62,2 & 126 & 48,5 & 80 & 43,5 \\
\hline \multicolumn{9}{|c|}{ Início ou retomada das atividades religiosas } \\
\hline Sim & 46 & 38,3 & 70 & 45,2 & 39 & 46,4 & 57 & 53,3 \\
\hline Não & 74 & 61,7 & 85 & 54,8 & 45 & 53,6 & 50 & 46,7 \\
\hline
\end{tabular}

Fonte: elaboração própria.

em cada município. A etnia e o nível de escolaridade dos participantes do estudo em cada município são semelhantes com a caracterização populacional de cada região 15 . Contudo, são aspectos que não devem ser encarados com naturalidade frente à necessidade de promoção da equidade na distribuição de serviços e recursos nas diferentes regiões do país.

Apesar do recorte do estudo não mensurar o grau de adequação do tratamento, em relação ao seu período de realização e tipos de intervenções realizadas, a pesquisa permitiu identificar um evento determinante do percurso de cuidados em saúde mental: o momento de identificação inicial do problema de saúde mental por um profissional de saúde. Nesse sentido, entre os fatores que podem contribuir para uma alta presença da $\mathrm{AB}$ na identificação inicial dos casos em São Paulo (54,3\%) está a presença de médicos psiquiatras em seus NASF. Já em Porto Alegre, onde 37\% dos casos foram identificados na $\mathrm{AB}$, vale ressaltar, além da presença de psiquiatras em algumas equipes de NASF, que o encaminhamento aos CAPS no período da pesquisa era fortemente regulado por este Núcleo, funcionando como gatekeeper da AE em saúde mental. Um resultado que ilustra essa perspectiva é a baixa proporção em Porto Alegre de usuários cuja identificação inicial do problema de saúde mental ocorreu no CAPS (8\%). Já no Município de Campinas, onde os serviços operam na modalidade "porta aberta”, a identificação no CAPS chegou a 25,5\% dos casos.

De forma dissonante ao preconizado por órgãos internacionais 16 e outros autores 17 como uma boa prática no cuidado de saúde mental, foi observada ainda uma forte presença de equipamentos hospitalares - gerais e psiquiátricos - como locais de identificação inicial do problema de saúde mental. Esse aspecto pode estar relacionado com uma baixa capacidade da $\mathrm{AB}$ em identificar e realizar o manejo dos casos em tempo oportuno 18,19. Nesse sentido, cabe pontuar a necessidade de fortalecer essa porta de entrada para qualificar a atenção em saúde mental.

Em Fortaleza, 36,7\% dos casos de saúde mental haviam sido identificados pela primeira vez em hospitais psiquiátricos. Ressalta-se que o período anterior à realização da pesquisa havia sido marcado por uma considerável redução de psicólogos nas equipes NASF do município, apontando indícios de como a falta de investimentos neste ponto de atenção afeta o aumento da demanda em equipamentos de maior complexidade.

Destaca-se que o NASF é essencial para fomentar cuidado colaborativo por meio do matriciamento dos casos entre a $\mathrm{AB}$ e os serviços especializados. $\mathrm{O}$ papel das equipes de apoio nessa articulação tem sido objeto de numerosos estudos no Brasil e no exterior 19,20,21. Contudo, os resultados deste trabalho apontam a necessidade de avançar a avaliação do impacto das diferentes configurações do NASF e equipes de apoio dentro das redes de saúde, especialmente no que diz respeito aos impactos da função de gatekeeper. 
A garantia de prescrição e acesso adequados aos medicamentos psicotrópicos é um dos pontos fundamentais para a superação da lacuna de cuidado à população com transtornos mentais, devendo ocorrer concomitantemente às ações de fomento à inserção e reabilitação psicossocial 5,22. Sendo assim, torna-se preocupante a baixa proporção de usuários que recebiam prescrição de medicamentos na AB no Município de Fortaleza (31,3\%), somada aos episódios de indisponibilidade das medicações prescritas na farmácia utilizada pelos usuários, nos seis meses que antecederam a aplicação do questionário $(71,9 \%)$.

A importância da dispensação e uso responsável de psicofármacos na assistência à saúde mental tem abundantes evidências na literatura, sendo destacado o efeito nocivo da retirada abrupta da medicação 23 . Essa perspectiva ressalta a gravidade da descontinuidade na oferta medicamentosa ainda presente nas redes de saúde, dificultando também a aderência e o compartilhamento das decisões em relação ao tratamento farmacológico 24,25,26.

No que diz respeito à reabilitação psicossocial, foram encontrados resultados positivos em todos os municípios acerca do início ou retomada das atividades de lazer e religião. Contudo, resultados mais discretos foram encontrados no que se refere ao início ou retomada das atividades de trabalho, cujas prevalências oscilaram entre 17,3\% (São Paulo) e 39,6\% (Campinas).

Esses resultados corroboram os achados de um estudo nos CAPS no sul do Brasil, que identificou maior efetividade dos serviços no estímulo às atividades de lazer e socialização, em detrimento do estímulo à inserção no mercado de trabalho 13 . Outro estudo aponta que apenas $18 \%$ dos usuários de CAPS ou hospital psiquiátrico possuem algum tipo de trabalho remunerado, ao passo que $39 \%$ recebem benefícios previdenciários ou auxílios assistenciais e 38\% não têm renda própria 27.

Em 2016, transtornos mentais foram a quarta causa mais frequente de concessão de auxílio-doença e representaram a maior quantidade de aposentadorias por invalidez em vigência 28 . A alta taxa nacional de desemprego, o déficit por vezes apresentado nas habilidades profissionais de pessoas com transtorno mental grave, assim como o preconceito em processos seletivos 27 , tornam os benefícios previdenciários ou auxílios assistenciais muitas vezes os únicos caminhos possíveis de sobrevivência econômica, sendo ainda bastante incipientes as iniciativas de geração de renda e trabalho protegido 29 .

As redes assistenciais de saúde mental devem ser orientadas por critérios éticos e clínicos 30,31. Sendo assim, este trabalho contribui para a discussão do panorama brasileiro ao trazer novas evidências sobre a persistência da desigualdade no contexto nacional, apontando algumas configurações das redes assistenciais em saúde mental que poderiam sofrer ajustes visando ao melhor desempenho dos serviços e aprimoramento do seguimento longitudinal dos indivíduos com transtornos mentais.

Cabe destacar que este estudo tem algumas limitações que restringem a extrapolação dos achados. Não foi possível a inclusão de municípios das regiões Norte e Centro-oeste do país como campos de pesquisa, aspecto que poderia ter dado maior profundidade à análise das desigualdades regionais.

Dada a aleatorização da amostra por tempo de recrutamento e não por métodos probabilísticos, os dados podem estar sujeitos a vieses de seleção. Contudo, frente à escassez de estudos abrangentes quanto à avaliação das redes de cuidado em saúde mental no país, este trabalho pode configurar um importante norteador na formulação de questões e desenhos de pesquisa mais complexos e melhor alinhados com as heterogeneidades vivenciadas no sistema de saúde brasileiro.

\section{Conclusão}

Frente às importantes disparidades em saúde vivenciadas pelos usuários com transtornos mentais, por meio deste estudo foi possível lançar luz sobre fragilidades e potencialidades do funcionamento das redes de saúde em quatro grandes municípios brasileiros. Valendo-se de uma abordagem mista, que buscou enriquecer a interpretação dos dados quantitativos de questionários com a descrição qualitativa das redes de saúde dos municípios estudados, foi possível trazer à tona a complexidade desses sistemas, bem como as heterogeneidades apresentadas por eles.

A despeito da expressiva expansão de serviços especializados de saúde mental, vivenciada no país nas duas últimas décadas, a assistência em saúde mental ainda apresenta importantes desigualdades regionais que se manifestam tanto na diferença entre o perfil da população atendida em cada região quanto no desempenho da rede de cuidado no processo de reabilitação psicossocial dos usuários. 
Entre os aspectos que se destacaram, pontua-se a persistência de serviços de alta complexidade (hospitais) como local de identificação dos problemas de saúde mental, além da baixa proporção de usuários que receberam tratamento de saúde mental na AB. Dessa forma, evidencia-se a necessidade de fortalecimento desse ponto de atenção como local de identificação - preferencialmente precoce - e manejo dos transtornos mentais.

A assistência farmacêutica aos usuários com transtornos mentais também foi identificada como um desafio para as redes de saúde estudadas. Destaca-se que a prescrição de medicamentos psicotrópicos na $\mathrm{AB}$ ainda é baixa, especialmente nos municípios de Campinas e Fortaleza. Os resultados acerca dessa dimensão apontaram ainda para problemas quanto à continuidade do tratamento medicamentoso, que tem sido desfavorecida particularmente em Fortaleza e Porto Alegre, pela falta dos medicamentos prescritos na farmácia utilizada pelos usuários.

O estudo evidenciou ainda que os resultados relacionados ao início ou retomada das atividades de trabalho por parte dos usuários após a sua entrada nos serviços especializados de saúde mental ainda são baixos, em especial quando comparados às atividades de lazer e religião.

\section{Colaboradores}

C. E. M. Amaral, C. A. S. Treichel e R. T. OnockoCampos contribuíram com a concepção e projeto do estudo, análise e interpretação dos dados, redação e revisão crítica relevante do conteúdo intelectual. P. M. S. B. Francisco contribuiu com a análise e interpretação dos dados e revisão crítica relevante do conteúdo intelectual. Todos os autores aprovaram a versão final a ser publicada e são responsáveis por todos os aspectos do trabalho na garantia da exatidão e integridade de qualquer parte da obra.

\section{Informações adicionais}

ORCID: Carlos Eduardo Menezes Amaral (00000002-1719-1080); Carlos Alberto dos Santos Treichel (0000-0002-0440-9108); Priscila Maria Stolses Bergamo Francisco (0000-0001-7361-9961); Rosana Teresa Onocko-Campos (0000-0003-04695447).

\section{Agradecimentos}

Os autores agradecem o apoio financeiro do Ministério da Ciência e Tecnologia e do Conselho Nacional de Desenvolvimento Científico e Tecnológico (CNPq) - Edital MCTI/CNPq/CT Saúde/MS/ SCTIE/Decit no 41/2013.

\section{Referências}

1. Mnookin S. Out of the shadows: making mental health a global development priority. http://documents.worldbank.org/curated/ en/270131468187759113/Out-of-the-shad ows-making-mental-health-a-global-develop ment-priority (acessado em 30/Mai/2019).

2. World Health Organization. Global mental health: how are we doing? http://www.who. int/mental_health/evidence/atlas/interactive_ infographic_2015.pdf?ua=1 (acessado em 30/ Mai/2019).

3. World Health Organization. Comprehensive mental health action plan 2013-2020. Geneva: World Health Organization; 2013.

4. Mari JJ, Thornicroft G. Princípios que deveriam nortear as políticas de saúde mental em países de baixa e média rendas. Rev Bras Psiquiatr 2010; 32:212-3.

5. Desviat M. Coabitar a diferença: da reforma psiquiátrica à saúde mental coletiva. São Paulo: Zagodoni; 2018.

6. Coordenação Geral de Saúde Mental, Álcool e Outras Drogas, Departamento de Ações Programáticas Estratégicas, Secretaria de Atenção à Saúde, Ministério da Saúde. Saúde mental no SUS: cuidado em liberdade, defesa de direitos e rede de atenção psicossocial. Relatório de gestão 2011-2015. Brasília: Ministério da Saúde; 2016. 
7. Rodriguez J. Los servicios de salud mental en América Latina y el Caribe: la evolución hacia un modelo comunitário. Rev Psiquiatr Urug 2011; 75:86-96.

8. Borysow IC, Furtado JP. Access, equity and social cohesion: evaluation of intersectoral strategies for people experiencing homelessness. Rev Esc Enferm USP 2014; 48:1066-73.

9. Onocko-Campos RT, Amaral CEM, Saraceno B, Oliveira BDC, Treichel CAS, Delgado PGG. Atuação dos Centros de Atenção Psicossocial em quatro centros urbanos no Brasil. Rev Panam Salud Pública 2018; 42:e113.

10. Creswell JW. Research design: qualitative, quantitative, and mixed methods approaches. Thousand Oaks: SAGE; 2009.

11. Onocko-Campos RT, Campos GWS, Amaral CEM, Tanaka OY. Apontamentos para estudo de sistemas de saúde: análise multifacetada e agravos traçadores. Rev Saúde Pública 2020; 54:102.

12. Tomasi E, Facchini LA, Piccini RX, Thumé E, Silva RA, Gonçalves H, et al. Efetividade dos centros de atenção psicossocial no cuidado a portadores de sofrimento psíquico em cidade de porte médio do Sul do Brasil: uma análise estratificada. Cad Saúde Pública 2010; 26:80715 .

13. Franzmann UT, Kantorski LP, Jardim VMR, Treichel CAS. Estudo das mudanças percebidas em usuários de Centros de Atenção Psicossocial do Sul do Brasil a partir de sua inserção nos serviços. Saúde Debate 2018; 42 (n.spe 4):166-74.

14. Kantorski LP, Jardim VMR, Treichel CAS, Demarco DA, Neutzling AS, Oliveira MM, et al. Satisfaction with mental health community services among patients' relatives. Rev Bras Epidemiol 2017; 20:237-46.

15. Instituto Brasileiro de Geografia e Estatística. Censo demográfico - 2010. http://www. ibge.gov.br/home/estatistica/populacao/cen so2010/default.shtm (acessado em 30/Mai/ 2019).

16. World Health Organization. mhGAP intervention guide for mental, neurological and substance use disorders in non-specialized health settings. Geneva: World Health Organization; 2010.

17. Thornicroft G, Tansella M. What are the arguments for community-based mental health care? Copenhagen: WHO Regional Office for Europe; 2003.

18. Campos RTO, Gama CA, Ferrer AL, Santos DVD, Stefanello S, Trapé TL, et al. Saúde mental na atenção primária à saúde: estudo avaliativo em uma grande cidade brasileira. Ciênc Saúde Colet 2011; 16:4643-52.
19. Athié K, Menezes AL, Silva AM, Campos M, Delgado PG, Fortes S, et al. Perceptions of health managers and professionals about mental health and primary care integration in Rio de Janeiro: a mixed methods study. BMC Health Serv Res 2016; 30:532.

20. Macinko J, Lima-Costa MF. Horizontal equity in healthcare utilization in Brazil, 1998-2008. Int J Equity Health 2012; 11:33.

21. Fortes S, Menezes A, Athié K, Chazan LF, Rocha $\mathrm{H}$, Thiesen J, et al. Psiquiatria no século XXI: transformações a partir da integração com a atenção primária pelo matriciamento. Physis (Rio J.) 2014; 24:1079-102.

22. World Health Organization. mhGAP: Mental Health Gap Action Programme. Scaling up care for mental, neurological and substance use disorders. Geneva: World Health Organization; 2008.

23. Breggin PR. Psychiatric drug withdrawal: a guidebook for prescribers, therapists, patients, and their families. New York: Springer; 2012.

24. Fenton WS, Blyler CR, Heinssen RK. Determinants of medication compliance in schizophrenia: empirical and clinical findings. Schizophr Bull 1997; 23:637-51.

25. Moncrieff J. The myth of the chemical cure: a critique of psychiatric drug treatment. Nova York: Macmillan; 2008.

26. Del-Barrio LR, Campos RTO, Stefanello S, Santos DVD, Cyr C, Benisty L, et al. Human rights and the use of psychiatric medication. J Public Ment Health 2014; 13:179-88.

27. Assunção AA, Lima EP, Guimarães MDC. Transtornos mentais e inserção no mercado de trabalho no Brasil: um estudo multicêntrico nacional. Cad Saúde Pública 2017; 33:e00166815.

28. Ministério da Previdência Social. Anuário estatístico da previdência social - 2017. Brasília: Ministério da Previdência Social; 2017.

29. Morato GG, Lussi IAO. Caracterização de iniciativas de geração de trabalho e renda destinadas a usuários de serviços de saúde mental e aproximação com a economia solidária: a realidade do Estado de São Paulo. Rev Ter Ocup 2015; 26:336-44.

30. Campos RTO, Costa M, Pereira MB, Ricci EC, Enes GCT, Chavez LJE, et al. Recovery, citizenship, and psychosocial rehabilitation: a dialog between Brazilian and American mental health care approaches. Am J Psychiatr Rehabil 2017; 20:311-26.

31. Thornicroft G, Tansella M. Boas práticas em saúde mental comunitária. São Paulo: Manole; 2010. 
Abstract

This study aimed to assess characteristics of healthcare networks in four large Brazilian cities (Campinas, Fortaleza, Porto Alegre, and São Paulo), in the provision of mental healthcare. The following outcomes were used: (i) place of identification of the mental health problem; (ii) mental healthcare in primary care; (iii) pharmaceutical care in mental health; and (iv) social rehabilitation. This is a mixed-methods study with a concurrent and sequential approach, conducted with 10 administrators and 1,642 users of Centers for Psychosocial Care (CAPS, in Portuguese) in the four cities. The study showed the persistence of high-complexity services such as hospitals as the site for initial identification of mental health problems in Campinas (40\% of users) and Fortaleza (37\%); low proportion of mental health treatment in primary care (Fortaleza, 23\%); differences between cities in psychotropic medication prescription in primary care (Porto Alegre, 68\%; São Paulo, 64\%; Campinas, 39\%; Fortaleza, 31\%) and in shortages of prescribed medication (higher in Fortaleza, 58\%; lower in Campinas, 28\%); and overall frailty in enabling return to work (lower in São Paulo, 17\%; higher in Campinas, 39\%), with better overall results regarding religion and leisure activities (higher in São Paulo, 53\% and $56 \%$, respectively). The study contributes to the discussion of the Brazilian scenario of mental healthcare, with evidence of persistent inequalities in the national context, pointing to gaps in some mental healthcare network configurations with the potential for better performance and longitudinal follow-up.

Primary Care; Secondary Care; Community Mental Health Services; Delivery of Health Care; Evaluation Methodology

\section{Resumen}

El objetivo de este estudio fue evaluar características de las redes de salud en cuatro grandes municipios brasileños (Campinas, Fortaleza, Porto Alegre y São Paulo), en lo que se refiere prestación de cuidados en salud mental. Se utilizaron como resultados: (i) lugar de identificación del problema de salud mental; (ii) atención en salud mental en la atención básica; (iii) asistencia farmacéutica en salud mental; y (iv) reinserción social. Se trata de un estudio analítico con métodos mixtos, de enfoque concomitante y secuencial, realizado con 10 gestores y 1.642 usuarios de Centros de Atención Psicosocial (CAPS) de los municipios citados. Se observó la persistencia de servicios de alta complejidad, tales como hospitales, respecto al lugar de identificación inicial del problema de salud mental en Campinas ( $40 \%$ de los usuarios) y Fortaleza (37\%); baja proporción de tratamiento de salud mental en la atención básica (Fortaleza, 23\%); hubo diferencias entre los municipios en lo que se refiere a la prescripción de medicamentos psicotrópicos en la atención básica (Porto Alegre, 68\%; São Paulo, 64\%; Campinas, 39\%; Fortaleza, 31\%), así como en la falta de medicamentos prescritos (mayor en Fortaleza, 58\%; menor en Campinas, 28\%); y fragilidad en general en el retorno al trabajo (menor en São Paulo, 17\%; mayor en Campinas, 39\%), existiendo mejores resultados en general, en relación con la religión y ocio (mayores en São Paulo, 53\% y 56\%, respectivamente). Se trata de un estudio que contribuye a la discusión del panorama brasileño de la asistencia en salud mental, con evidencias de la persistencia de desigualdades en el contexto nacional, además apunta lagunas en algunas configuraciones de las redes de salud mental con potencial para un mejor desempeño y seguimiento longitudinal.

Atención Básica; Atención Secundaria de Salud; Servicios Comunitarios de Salud Mental; Prestación de Atención de Salud; Métodos de Evaluación
Recebido em 06/Mar/2020

Versão final reapresentada em 03/Ago/2020

Aprovado em 05/Ago/2020 